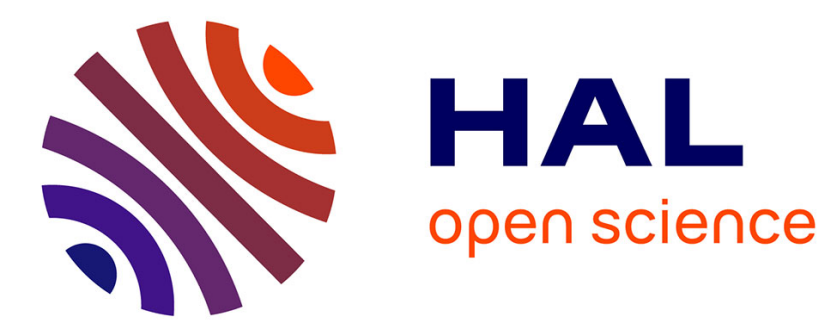

\title{
Parallel microrobot actuated by capillary effects.
}

\author{
Cyrille Lenders, Michaël Gauthier, Pierre Lambert
}

\section{To cite this version:}

Cyrille Lenders, Michaël Gauthier, Pierre Lambert. Parallel microrobot actuated by capillary effects.. IEEE International Conference on Robotics and Automation, ICRA'11., May 2011, Shanghai, China. pp.6015-6020. hal-00648640

\section{HAL Id: hal-00648640 \\ https://hal.science/hal-00648640}

Submitted on 6 Dec 2011

HAL is a multi-disciplinary open access archive for the deposit and dissemination of scientific research documents, whether they are published or not. The documents may come from teaching and research institutions in France or abroad, or from public or private research centers.
L'archive ouverte pluridisciplinaire HAL, est destinée au dépôt et à la diffusion de documents scientifiques de niveau recherche, publiés ou non, émanant des établissements d'enseignement et de recherche français ou étrangers, des laboratoires publics ou privés. 


\title{
Parallel Microrobot Actuated by Capillary Effects
}

\author{
Cyrille Lenders, Michaël Gauthier and Pierre Lambert
}

\begin{abstract}
This paper presents a new actuation mean for a parallel microrobot based on capillary effect, combining surface tension and pressure effects. The device presented is a compliant moving table having 6 degrees of freedom (dof) among which three are actuated: $z$ axis translation having a stroke of a few hundreds of microns, and $\theta_{x}$ and $\theta_{y}$ tilt angles up to about $15^{\circ}$. The structure is immersed in a liquid and the actuation principle is based on fluidic parameters (pressure and volume). A model to calculate the stiffness of the system is presented and validated by experimental measurements. Some issues inherent to this type of actuation are also addressed. The presented device is an illustration of a promising solution for microrobotic actuation using capillary effects in a liquid media.
\end{abstract}

\section{INTRODUCTION}

The application field of the microrobot proposed in this article is to offer compliant micropositioning system for micro-assembly operations. Micro-assembly, which deals with the assembly of submillimetric components, consists in gripping, moving, placing and releasing microcomponents at defined locations. The major complexity in microassembly is coming from the fact that the surface forces inducing significant perturbations on micro-object positioning are highly dependent on the environment [24]. Comparative analysis has shown that a liquid environment is able to reduce the level of perturbations compared to manipulation in the air [11]. Indeed adhesion force and electrostatic force can be reduced and the increase of the dynamic viscosity is able to stabilize the behavior of the micro-object. It opens the way for new microhandling and microactuating solutions. For example, submerged ice microgripper able to release object in liquid without adhesion perturbations has been proposed by LopezWalle [21], [22], [20]. Dielectrophoresis or optical non contact manipulation usually used with biological cells can also be used to control the position of the microcomponents in liquid [2], [12], [13]. Recent works has also shown that the modification of the chemical properties of the medium (e.g. $\mathrm{pH}$ ) is able to change the adhesion properties of objects from an adhesive behavior to a repulsive one [6], [7]. In that case, chemical stimuli could be used to directly control the grasping and the release of the objects with a monodigital gripper [8]. Beyond microhandling, micro-assembly in liquid requires also micropositioning stages which is the framework of this article. We are proposing a microrobot principle acting

C. Lenders and P. Lambert are with Faculty of Applied Sciences, Bio Electro and Mechanical Systems Department, Université libre de Bruxelles (U.L.B.), Av. F.D. Roosevelt 50, 1050 Bruxelles, Belgium (e-mail: clenders@ulb.ac.be, plambert@ulb.ac.be)

M. Gauthier and P. Lambert are with the FEMTO-ST Institute, AS2M Department, 24 rue Savary, 25000 Besançon, France (e-mail: michael.gauthier@femto-st.fr) in liquid whose principle is based on surface tensions induced by air microbubbles.

Surface tension based actuation are very efficient at microscale because of favorable scaling effects. This actuation mean has been used as a gripping principle [15], and as a driving principle for a micromotor [27].

\section{DEVICE OVERVIEW}

The device presented here is a compliant table that can be used to perform microrobotic assembly tasks in liquid environment. The table has six degrees of freedom (DOF), among which three are actuated: the translation along the direction orthogonal to the platform plane $(\bar{z})$, and two rotations along axes parallel to the platform plane.

The device is made out of three main components: the moving table, the three bubbles which are used as compliant actuators, and the platform from which the bubbles are generated (Fig. 1).

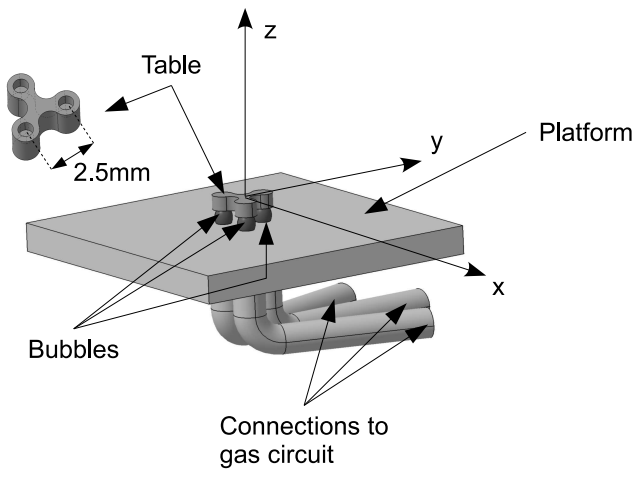

Fig. 1. Schematic view of the device, composed of a compliant table lying on 3 bubbles generated from a platform

Bubbles are the key of this device. Thanks to surface tension at the gas-liquid interface, and to the compressibility of the gas, a microbubble can be used as a compliant actuator. Indeed, the force developed by a bubble sandwiched between two solids has two components: one due to surface tension along the triple line, and one due to the pressure difference across the interface. If the contact angles in the liquid are smaller than $90^{\circ}$, the mean curvature of bubble interface is positive, and the pressure inside the bubble is larger than in the surrounding liquid. When the bubble is squeezed, the bubble is compressed and exerts a repulsive force on the solids. When the bubble is stretched, the bubble exerts an attractive force on the solids. Its behavior is comparable to a spring, from a mechanical point of view. But we will demonstrate that a bubble presents several advantages 
compared to classical mechanical springs. One of these advantages is that it is possible to actuate the bubbles using fluidic parameters $(P, V)$.

To be efficient, it is necessary to control the generation of the bubbles. The best way is to use a system derived from a syringe pump: the idea is to vary the volume of a tank containing gas in order to push the gas out of a hole. This principle has been developed in [19], in which it is underlined that surface tension must be taken in account in the bubble generator design. Some authors propose other principles for bubble generators, such as electrolysis [3], [4], [5], [23], [17], [28] or temperature rise [1], [9], [10], [16], [26], but the control of bubble size is more complex with these methods.

\section{Design And MANUfACtURING}

When designing the device, the weight of the table should be of course considered, since it will use some of the bubble compliance to reach equilibrium. Consequently, the table has a trefoil shape (Fig.1) in order to reduce the weight of the platform.

But more important is the anchoring of the bubbles on the platform and on the table. The anchoring is a mean to ensure the bubbles will remain at a specific location even under mechanical stress. Two methods can be used to ensure this anchoring: a mechanical method and a chemical method [25]. In both cases, the idea is to create an energetic barrier preventing the triple line to move. The mechanical method consists in creating a sudden variation in the solid part profile: the contact angle $\theta$ must increases up to an angle called advancing angle value before the triple line can move. The chemical method consists in the deposition of a coating having a different surface energy. Here again, a movement of the triple line requires a change of the contact angle at the borderline of the two surfaces with different energy.

Another advantage for bubbles is that they induce the automatic centering of the table. Because of energy minimization, bubbles tend to have the smallest possible interface surface. Henceforth, the bubbles will always move the table in such a way they are in the most favorable position. If the table and platform anchoring means have the same layout, the table will automatically centers above the platform to superpose both layouts. The assembly of the table with the rest of the device is therefore very easy.

The presented prototype is made out of aluminum and has been manufactured using a conventional CNC milling machine. In this design, the anchoring has been realized using the mechanical method. Figure 2 (a) shows the anchoring in this first design. This method has the advantage of being very simple. However, there is a more efficient configuration, illustrated on Fig. 2 (b), but this configuration could not be manufactured using conventional machining processes. We have already started investigating to find other manufacturing means. One promising method is to use an excimer laser to machine polycarbonate.

The bubble generator is based on a volume controlled gas injection principle, like a syringe pump. In the prototype, the bubble generator is a flexible hose containing the gas,

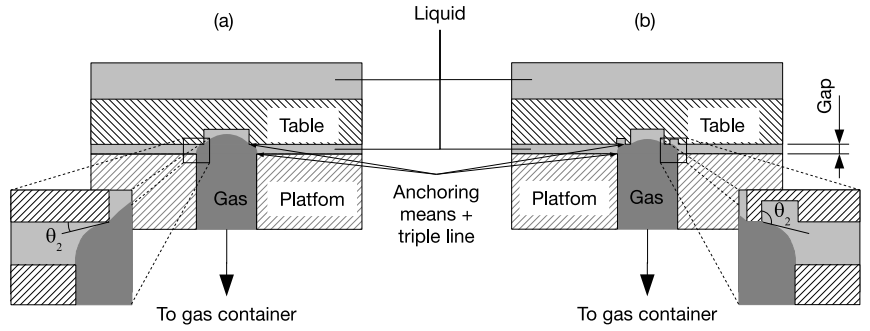

Fig. 2. Illustration of one leg of the table. A bubble is generated from the platform and lift the table. The anchoring means are in this case geometrical changes that improves the fixation of the line where solid-liquid and gas touches each other (triple line)

which is squeezed to generate the bubble. The volume of gas initially contained in the hose can be adjusted by filling the hose with an incompressible fluid (Fig. 3).

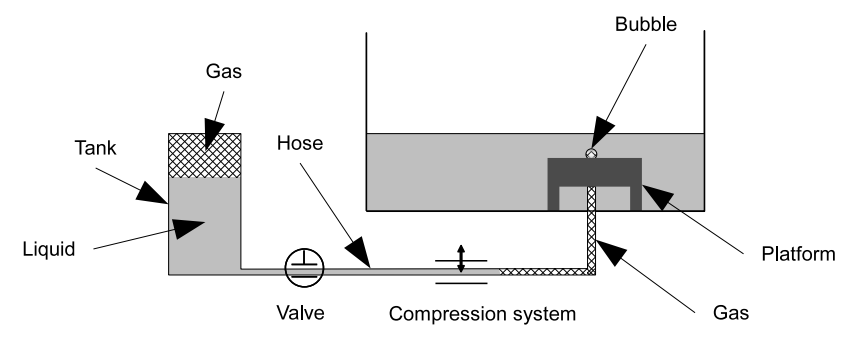

Fig. 3. Illustration of volume controlled bubble generator for the prototype. A bubble is grown by squeezing the flexible hose joining the platform and a container

\section{EQUATIONS}

The ultimate purpose of this paper is to find the dynamic equation to predict the movement of the table under mechanical stress. We propose to model the dynamic of the table as a mass-spring-dashpot system. The general equations for actuated movements are:

$$
\begin{aligned}
m \ddot{h}+b_{t} \dot{h}+k_{t} h & =R_{z} \\
I_{x, y} \alpha_{x, y}+b_{r} \alpha_{x, y}^{\dot{*}}+k_{r} \alpha_{x, y} & =\Gamma_{x, y}
\end{aligned}
$$

where $m$ is the mass of the table, $h$ is the gap between the platform and the table, $b_{t}$ is the viscous friction coefficient, $k_{t}$ is the stiffness coefficient, $R_{z}$ is the force resultant component along the axis orthogonal to the platform ( $\mathrm{z}$ axis), $I_{x, y}$ is the moment of inertia along $x$ and $y$ axes, $\alpha_{x, y}$ is the rotation angle in the direction $x$ and $y, b_{r}$ is the viscous coefficient for rotation movement, $k_{r}$ is the rotation stiffness coefficient, and $\Gamma_{x, y}$ is the torque resultant along $x$ and $y$ direction.

The objective is to find values for $k_{t}, b_{t}, k_{r}$ and $b_{r}$. In this paper, we will focus on the modeling of $k_{t}$.

To determine the stiffness of the table, we have to determine the stiffness of one bubble. The total stiffness can be seen as the sum of the stiffness for each bubble, since the configuration is like three parallel springs.

To find the stiffness of a single bubble, we have to model the force developed by a bubble as a function of the distance between the table and the platform. This model takes account 
of the total volume in the bubble generator gas circuit $V$, the surface tension at the fluid-gas interface $\gamma$, the pressure in the liquid outside the bubble $P_{0}$, the temperature $T$, and the geometry of the anchoring means.

We suppose that the anchoring means are circular, and the table is placed above the platform in such a way that the bubbles have an axisymmetric shape.

Laplace law relates the surface tension $\gamma$, the pressure difference across the interface $\Delta P=P_{\text {in }}-P_{\text {out }}$, and the mean curvature of the interface $H$ :

$$
\Delta P=2 \gamma H
$$

If the height of the bubble is smaller than the capillary length, the variation of hydrostatic pressure along $z$ axis is negligible. Therefore, the mean curvature is uniform along $z$ axis. The equation of interface profile $r(z)$ is given by:

$$
\frac{\Delta P}{\gamma}=2 H=-\frac{\frac{\partial^{2} r}{\partial z^{2}}}{\left(1+\left(\frac{\partial r}{\partial z}\right)^{2}\right)^{\frac{3}{2}}}+\frac{1}{r\left(1+\left(\frac{\partial r}{\partial z}\right)^{2}\right)^{\frac{1}{2}}}
$$

The boundary conditions for this ODE are given by the bubble anchoring means, which impose $r$ at both ends of the profile. The value of the mean curvature is constrained by fluidic parameters. Indeed, Laplace equation (3) indicates that surface tension is responsible for a pressure variation in the bubble. Since the bubble is compressible, the pressure rise in the bubble will be responsible for a change of volume. Using gas law, it is possible to determine the new volume of gas in the system (volume in the bubble and volume in the gas container):

$$
\left(P_{0}+\Delta P\right) V=n R_{g} T
$$

The mean curvature $H$ must be chosen so that (5) agrees with the number of mole $n$ in the gas system. As a result there is a coupling between surface tension and gas compressibility.

When the geometry of the interface is found, it is possible to calculate the force generated by the bubble. As already mentioned, the force developed by a bubble has two components [14].

The surface tension force represents the tension in the interface. The force is the resultant of the distributed force tangent to the interface along the triple line. It is proportional to the sine of the contact angle, and to the surface tension:

$$
\overline{F_{T S}}=\oint_{\text {Triple line }} \gamma \sin (\theta) d l \bar{z}=\pi s \gamma \sin (\theta) \bar{z}
$$

where $s$ is the diameter of the anchoring circle and:

$$
\frac{1}{\tan (\theta)}=-\frac{\partial r}{\partial z}
$$

The pressure force represents the effect of the pressure difference across the bubble interface:

$$
\overline{F_{P}}=\iint_{\text {Solid/gas interface }} \Delta P d S \bar{z}=\pi \frac{s^{2}}{4} \Delta P \bar{z}
$$

The vertical force is therefore a function of the curvature, the surface tension and the gap. It is interesting to note that because the curvature is linked to the pressure by (3), the knowledge of gas mole number and pressure should therefore be sufficient to deduce the gap $(h)$ and the force applied to the table $(F)$ (Fig. 4). This is interesting because pressure measurement can be done anywhere in the gas circuit.
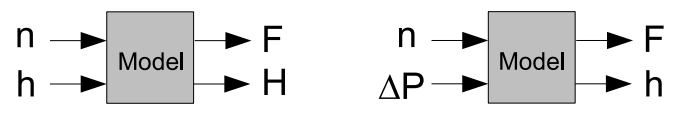

Fig. 4. Our model allows to find $H$ and $F$ assuming $n$ and $h$. This model can be inverted to find $F$ and $h$ from the knowledge of $n$ (known from setup configuration or calibration) and $\Delta P$, which may be measured anywhere in the circuit

The model detailed above was used to size up the prototype (Fig. 5). We first define the geometry of the anchoring circle. Then we assume a bubble having a certain height is generated from the platform. From these data, it is possible to define the distance between the table and the platform when they come into contact. Then we vary this distance and we search for the interface mean curvature $H$ ensuring a constant gas mole number $n$. Once $H$ is found for a value of the gap, it is possible to calculate the force generated by the bubble. Knowing the force for each gap value, it is possible to determine the stiffness of the bubble. Moreover, the model predicts that the volume of the gas circuit will have a significant influence on bubble stiffness.

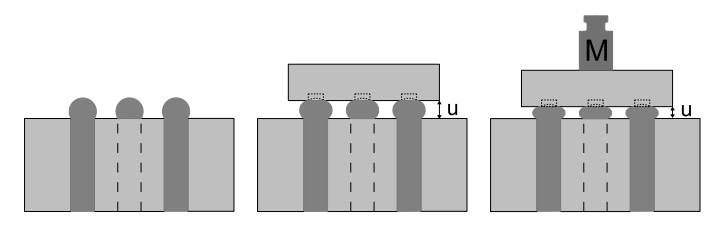

Fig. 5. Principle of the experimental validation of bubble stiffness model. First a single bubble is generated from each hole of the platform. Then the table is layed on the bubbles and the distance $u$ between the table and the platform is measured. Finally, different known masses are added successively and the evolution of $u$ is measured

This is illustrated in Fig. 6, where we have plotted the evolution of the force generated by a bubble as a function of the gap distance. There is a distance at which the force is zero, which is the distance at which bubble and table come into contact. The shape of the bubble is at this point a portion of a sphere. If the distance increases, we see that the force is negative, pulling the table towards the platform.

Another interesting property that can be demonstrated by this model is that for a given geometry (given anchoring position), the stiffness of the bubble is function of the volume in the gas circuit. If the volume increases, the bubble will be more compliant. If the volume is just large enough to generate one bubble, the behavior of the bubble will be 


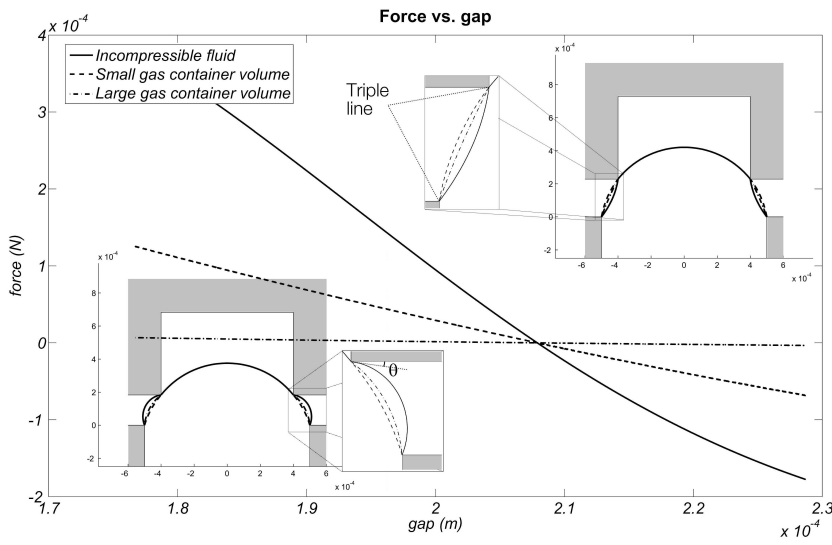

Fig. 6. Illustration of force vs. gap evolution for different gas system volume $\left(s_{\text {bottom }}=10^{-3} \mathrm{~m}, s_{\text {top }}=0.8 \cdot 10^{-3} \mathrm{~m}, \gamma=72 \cdot 10^{-3} \mathrm{Nm}^{-1}, h_{\text {init }}=\right.$ $0.4 \cdot 10^{-3} \mathrm{~m}$ and $P_{0}=101325 \mathrm{~Pa}$ ). If the gas container is large (here $10^{-6} \mathrm{~m}^{3}$ ), a small variation of volume will not change pressure significantly, so the pressure variation and bubble curvature variations are small. The stiffness of the bubble is in this case small. On the contrary, if the gas system volume is small (here $10^{-8} \mathrm{~m}^{3}$ ), any volume variation will induce a significant change in the gas pressure, and the shape of the bubble will vary significantly. The stiffness of the bubble is therefore larger. We have also indicated the case of an incompressible fluid, for which the stiffness is even larger. Both illustrations show the shape of the interface for the minimal (left) gap and maximal (right) gap

close to the one of an incompressible fluid, but will remain compliant because the interface can change its geometry. This is illustrated on Fig. 6 where we see that the order of magnitude for stiffness is $1 \mathrm{Nm}^{-1}$.

\section{EXPERIMENTAL RESULTS}

The model has been validated experimentally using the prototype. We first present a demonstration of the use of the prototype. Then we will apply mechanical loads on the table and compare the actual stiffness to model predictions.

\section{A. Demonstration of the Prototype}

We have manufactured and tested the prototype of the bubble carried compliant table. We have demonstrated that bubbles were able to withstand the force exerted by an aluminum table. We have also demonstrated that the table was compliant and able to move under an external solicitation. Finally, we have also demonstrated that it is possible to tilt the table or to move it in the vertical direction, by controlling the amount of gas injected in each bubble. All these results are illustrated in Fig. 7. The 3 actuated DOF are illustrated in Fig. 8.

\section{B. Validation of Vertical Force Model}

The model has been validated experimentally using the prototype. We used an image recording system to measure the gap, and we increased the force applied on the table by posing objects of known mass and density on the table. Figure 9 shows the measurement points and the corresponding model curve. Each measurement has been repeated 5 times, inducing some dispersion on the gap measured. Some deviation has been initially observed between the model and

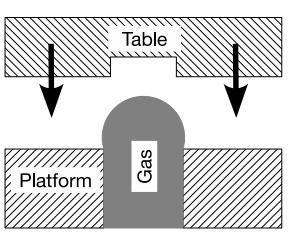

(a)
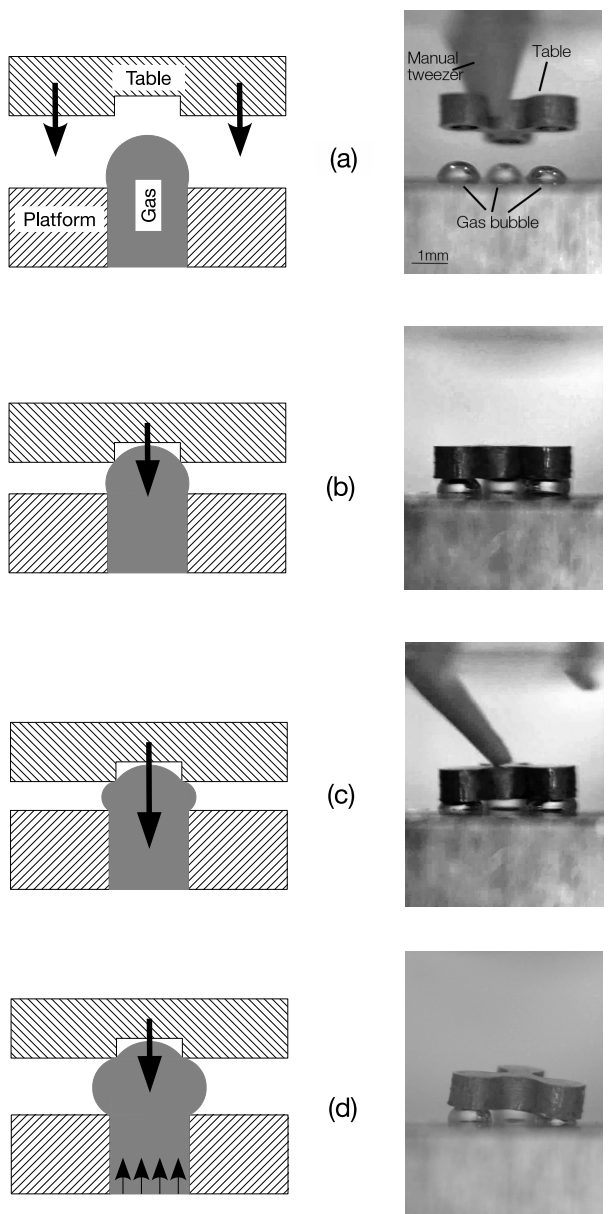

Fig. 7. Illustration of the capillary driven robotic table concept. Three bubbles are generated from the platform, and the table is dropped on the bubbles. The table is automatically self centered above the platform (a). To counterbalance the force exerted by the table, the bubbles change their shape as the gap distance reduces (b). If supplementary force is applied, the table moves down as the bubble generated force increases (c). It is also possible to lift or tilt the table by injecting more gas in the bubbles (d)

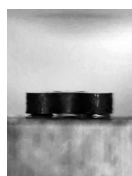

(a)

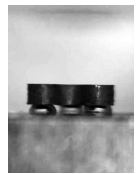

(b)

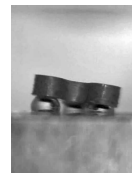

(c)

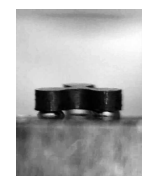

(d)
Fig. 8. View of the different degrees of freedom. (a) reference position, (b) vertical translation (here $\approx 250 \mu \mathrm{m}),(\mathrm{c}, \mathrm{d})$ rotations (here $\approx 10^{\circ}$ )

the experimental results. This is due to the hypothesis of having a constant number of gas moles, which is not satisfied. In order to best match the measurements by the model curve, we have assumed a linear diminution of $n$ with the gap, i.e. with the experiment time, of at maximum $0.5 \%$. We can conclude from these results that in these configurations, the vertical stiffness of a single bubble is about $1 \mathrm{Nm}^{-1}$. The stiffness of the entire table is 3 times this value. 


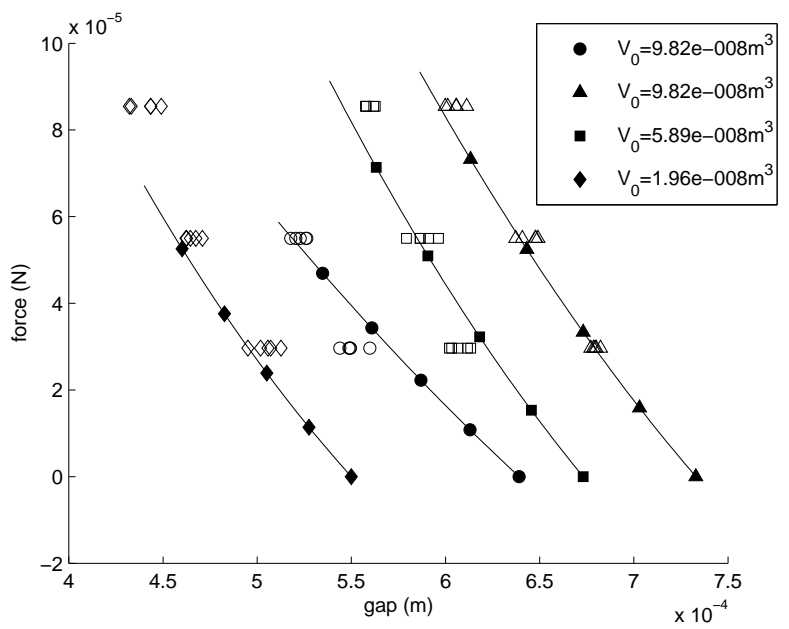

Fig. 9. Experimental validation of the vertical force model. The unfilled symbols represent the measurements, the filled symbols on curves represent the solution from the model corresponding to the experimental setup conditions

\section{Leak Tightness Issues}

There are two root causes for this decrease of gas mole in the circuit: the permeability of the flexible hoses made of silicone, and the gas dissolution in the liquid. A model of membrane permeability is given by [29]:

$$
P e=\frac{V_{g} d}{A t \Delta P}
$$

where $P e$ is the permeability of the material, $V_{g}$ is the volume of gas permeating through the material, $A$ is the area of the hose wall, equal to $\pi D l$ where $D$ is the inner diameter of the flexible and $l$ is the length of the hose filled with gas, $t$ is the time and $\Delta P$ is the pressure difference between inner and outer side of the hose. $P e$ is a function of the material, but also a function of the gas considered. For silicone, $P e=6510^{-9} \mathrm{~cm}^{2} \mathrm{~s}^{-1} \mathrm{cmHg}$ for $\mathrm{O}_{2}$ molecules, and $P e=2810^{-9} \mathrm{~cm}^{2} \mathrm{~s}^{-1} \mathrm{cmHg}$ for $\mathrm{N}_{2}$ molecules. For gas mixes, partial pressures must be used. For our application, if we suppose $\Delta P_{0_{2}}=0.2 \Delta P$ and $\Delta P_{\mathrm{N}_{2}}=0.8 \Delta P$, we can estimate the number of mole permeating through the hose wall.

To evaluate the decrease of mole number in the gas system, we have recorded a single bubble at the rate of 1 frame per second in water (length of hose filled with air: $0.3 \mathrm{~m}$, pressure drop estimated from first bubble and assumed constant: $186 \mathrm{~Pa})$. We represent some of the frames in Fig. 10. The same experiment has been realized with silicone oil (length of hose filled with air ${ }^{1}: 0.7 \mathrm{~m}$, pressure drop estimated from first bubble and assumed constant: $62 \mathrm{~Pa}$ ). We have plotted in Fig. 11 the evolution of mole number from experiments with water and oil, and the corresponding results due to the model of permeability. We see that the permeability explains the gas mole number variation in the case of oil, but does account only for a part of the variation in the case of water. With water, we suppose that the difference between model and experiment is due to air dissolution in the water.

\footnotetext{
${ }^{1}$ The remaining of the hose was filled with water
}

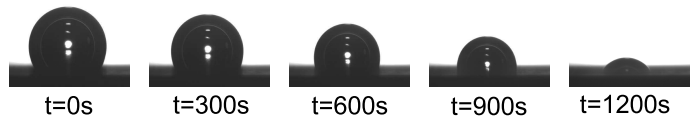

Fig. 10. Experiment to illustrate gas mole decrease over time in an airwater system

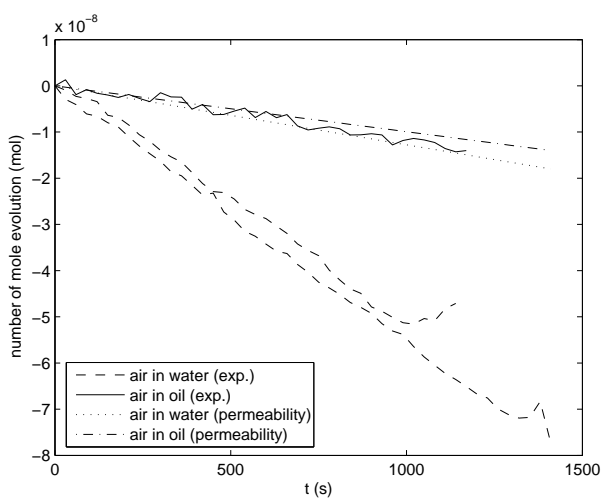

Fig. 11. Comparison of permeability model of gas permeation through flexible hoses with experiments. When the liquid is silicone oil, the permeability of the silicone hoses explains the origin of gas mole decrease in the system. When the liquid is water, only a part of the gas mole decrease is explained by the permeability of the silicone hose, the difference is probably due to gas dissolution in the water

To improve the device, it is therefore important to choose materials with a low permeability, such as PTFE instead of silicone. It is also important to prevent gas dissolution in the liquid. This can be done by either saturating the liquid with gas, or by using a liquid in which gas dissolution is weak, such as silicone oil. An improved version of the experimental setup has been built which gives promising results to avoid gas leakage (Fig. 13).

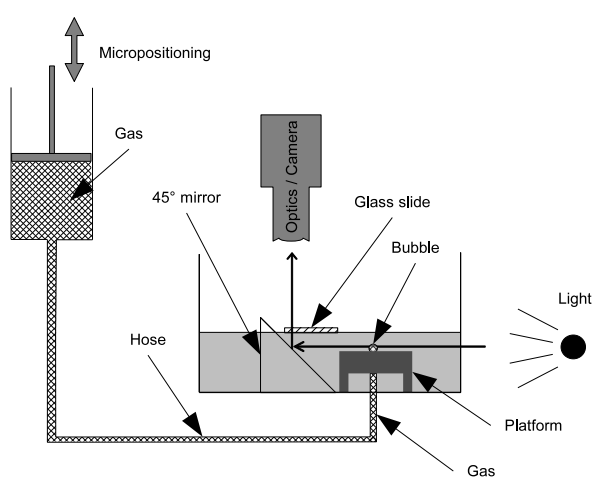

Fig. 12. Illustration of volume controlled bubble generator for the prototype. Three bubbles are grown by injecting gas from syringes actuated by a microposioning stage. The table is then deposited on the bubbles

\section{Vi. Conclusion and Perspectives}

We have presented in this paper a new design of compliant table, using the properties of gaseous bridges as compliant actuators. So far, we have validated the general concept and the model giving the stiffness of the table. There is still work to do to validate the other dynamic parameters. 


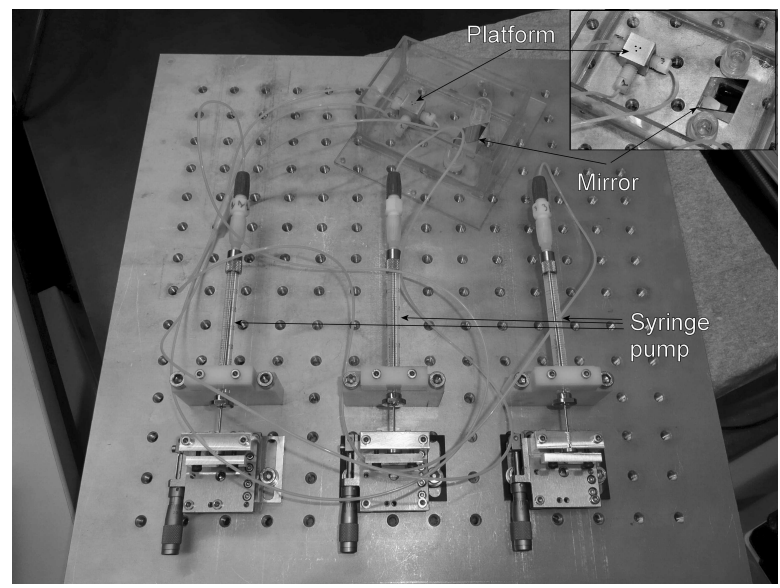

Fig. 13. View of an enhanced version of the test bed. The picture shows the manual micropositioning stages used as syringe pumps, and the test cell where the platform is immersed in a liquid. The illustrated version uses PTFE hoses and gas tight syringes

The lateral stiffness of a bubble must also be studied in order to predict the lateral forces that can be handled by the device. If the stiffness is too weak for the application, is it is possible to add bubbles in the system to push against the lateral faces of the table. Finally, some technological issues must be addressed. For example, we suppose in our model that the number of gas mole is constant. The model is very sensitive to this parameter and any gas dissolution in the liquid or any gas leak should be carefully avoided. The improvement of the anchoring sites must also be addressed using new manufacturing technologies.

\section{ACKNOWLEDGMENT}

The authors would like to thank the BRIC from Université libre de Bruxelles and PHC-Tournesol funding (WBI-FNRS) for their financial support.

\section{REFERENCES}

[1] Vladimir S. Ajaev, G. M. Homsy, and S. J. S. Morris. Dynamic response of geometrically constrained vapor bubbles. J. Colloid Interface Sci., 254:346-354, 2002.

[2] F. Arai, K. Onda, R. Litsuka, and H. Maruyama. Multi-beam laser micromanipulation of microtool by integrated optical tweezers. In Multi-beam laser micromanipulation of microtool by integrated optical tweezers, pages 2779-84, Kobe, Japan, 2009.

[3] H. Bouazaze, S. Cattarin, F. Huet, M. Musiani, and R.P. Nogueira. Electrochemical noise study of the effect of electrode surface wetting on the evolution of electrolytic hydrogen bubbles. J. Electroanalytical Chemistry, 597:60-68, 2006.

[4] Sang Kug Chung, Yuejun Zhao, and Sung Kwon Cho. On-chip creation and elimination of microbubbles for a micro-object manipulator. J. Micromech. Microeng., 18(9):095009 (13pp), 2008.

[5] Ron Darby and M. S. Haque. The dynamics of electrolytic hydrogen bubble evolution. Chem. Eng. Sci., 28:1129-1138, 1973.

[6] J. Dejeu, M. Gauthier, P. Rougeot, and W. Boireau. Adhesion forces controlled by chemical self-assembly and ph, application to robotic microhandling. ACS Applied Materials \& Interfaces, 1(9):1966-1973, 2009.

[7] J. Dejeu, P. Rougeot, M. Gauthier, and W. Boireau. Reduction of micro-object's adhesion using chemical functionnalisation. MicroNano Letters, 4(2):74-79, 2009.

[8] J. Dejeu, P. Rougeot, M. Gauthier, and W. Boireau. Robotic microhandling controlled by chemical self-assembly. In Proc. of the IEEE/RSJ Int. Conf. on Robotics and Intelligent Systems, St. Louis, Missouri, USA, Oct. 2009.
[9] Peigang Deng, Yi-Kuen Lee, and Ping Cheng. The growth and collapse of a micro-bubble under pulse heating. International Journal of Heat and Mass Transfer, 46:4041-4050, 2003.

[10] Peigang Deng, Yi-Kuen Lee, and Ping Cheng. An experimental study of heater size effect on micro bubble generation. International Journal of Heat and Mass Transfer, 49:2535-2544, 2006.

[11] M. Gauthier, S. Regnier, P. Rougeot, and N. Chaillet. Forces analysis for micromanipulations in dry and liquid media. Journal of Micromechatronics, 3(3-4):389-413, 2006.

[12] M. Kharboutly, M. Gauthier, and N. Chaillet. Modeling the trajectory of a micro-particle in a dielectrophoresis device. Journal of Applied Physics, 106:114312, dec 2009.

[13] M. Kharboutly, M. Gauthier, and N. Chaillet. Modeling the trajectory of a micro particle in a dielectrophoresis device. In IEEE International Conference on Robotics and Automation ICRA 2010, Alaska, USA May 2010.

[14] Pierre Lambert. Capillary Forces in Microassembly: Modeling, Simulation, Experiments, and Case Study. Microtechnology and MEMS. Springer, October 2007.

[15] Pierre Lambert, Frank Seigneur, Sandra Koelemeijer, and Jacques Jacot. A case study of surface tension gripping: the watch bearing. $J$. Micromech. Microeng., 16(7):1267-1276, 2006.

[16] Dmitri Lapotko and Ekaterina Lukianova. Laser-induced microbubbles in cells. International Journal of Heat and Mass Transfer, 48:227-234, 2005.

[17] S. Lee, W. Sutomo, C. Liu, and E. Loth. Micro-fabricated electrolytic micro-bubblers. International Journal of Multiphase Flow, 31:706722, 2005.

[18] Cyrille Lenders, Michaël Gauthier, and Pierre Lambert. Meniscussupported compliant table, 2009. patent request submitted: EP 09 172715.

[19] Cyrille Lenders, Michaël Gauthier, and Pierre Lambert. Microbubble generation using a syringe pump. In Proceedings of the 2009 IEEE International Conference on Intelligent Robots and Systems, SaintLouis (Missouri), October 11-15 2009.

[20] B. Lopez-Walle, M. Gauthier, and N. Chaillet. Principle of a submerged freeze gripper for micro-assembly. IEEE Trans. on Robotics, 24(4):897-902, 2008

[21] B. Lopez-Walle, M. Gauthier, and N. Chaillet. Dynamic modelling for a submerged freeze microgripper using thermal networks. Journal of Micromechanics and Microengineering, 20,(2):025001, 2010.

[22] B. Lopez-Walle, M. Gauthier, and N. Chaillet. Dynamic modelling for thermal micro-actuators using thermal networks. International Journal of Thermal Sciences, 49:2108-2116, 2010.

[23] Steven Lubetkin. The motion of electrolytic gas bubbles near electrodes. Electrochimica Acta, 48:357-375, 2002.

[24] S. Régnier M. Gauthier. Robotic micro-assembly. IEEE Press, 2010.

[25] M. Mastrangeli, S. Abbasi, C. Varel, C. Van Hoof, J.-P. Celis, and K.F. Bohringer. Self-assembly from milli- to nanoscales: methods and applications. Journal of Micromechanics and Microengineering, 19, 2009

[26] Abhijit Mukherjee and Satish G. Kandlikar. Numerical simulation of growth of a vapor bubble during flow boiling of water in a microchannel. Microfluid Nanofluid, (1):137-145, 2005.

[27] Atsushi Takei, Kiyoshi Matsumoto, and Isao Shomoyama. Capillary motor driven by electrowetting. LAB CHIP, 10:1781-1786, 2010.

[28] A. Volanschi, W. Olthuis, and P. Bergveld. Gas bubbles electrolytically generated at microcavity electrodes used for the measurement of the dynamic surface tension in liquids. Sensors and Actuators A, 52:18 22, 1996

[29] Haibing Zhang and Andy Cloud. The permeability characteristics of silicone rubber. In Proceedings of the 2006 SAMPE Fall Technical Conference "Global Advances in Materials and Process Engineering", Coatings and Sealants Section, Dallas, TX, November 2006. Society for the Advancement of Material and Process Engineering. 